\title{
Transient Analysis of a Complex Network of Gas Pipeline Compulsory in the Design by Dynamic Simulation
}

\author{
${ }^{1}$ Mohamed Chérif AbdelFettah TOUABTI* ${ }^{1}$ Messaoud GUELLAL \\ ${ }^{1}$ Laboratory of Chemical Engineering \\ University Ferhat Abbas, Faculty of Engineering, Sétif - Algeria
}

\begin{abstract}
Technical and economical calculations of pipelines networks of are made currently on the basis of stationary flow regimes that are to say of working regimes where the technical features (mainly flow speed and pressure) are supposed non variants with regard to the time. However the analysis of practical situations shows that the real flows are only unusually stationary.

The hypothesis of stationary aspect in the conception of pipeline networks is bound mainly to the mathematical difficulties describing the nonstationary out-flow in a complex network of pipelines: differential equation systems of non linear partial derivatives of very big dimension. However the analysis of practical situation shows that the real out-flows are only unusually stationary.

We suggest in this subject a modelling and simulation of a real pipeline with variable and transient regime and to study by means of numerical simulations the dynamic behavior of gas in pipeline by means of several scenario. The software used is the SIMONE of LIWACOM Group s.r.o and TGNET 5.6- E of SSI.
\end{abstract}

Keywords: Pipelines networks, gazodynamics, variable flow, modeling, simulation

\section{Introduction}

Traditionally, a new project of a system of gas pipeline starts with a step of technical and economic evaluation which takes into account the analysis of the stationary state of the flow with a multitude of assumptions such as intensification of the distribution market, minimal and maximum flow and the estimated number of compression stations. Once the economic and technical evaluation shows an interesting output of the project, the implied companies decide on its launching, and the step of the design starts. It is in this step that a transient analysis of the system appeared to be imperative.

\subsection{Why a transient analysis in the step of design? Off-line}

The first reason is related to the importance of the necessary investment for such a system constituted of pipelines and compressor stations. The conceived system has to be able to function according to different predictable scenarios. Otherwise the transport company would be penalized for the non-distribution of the agreed volume of gas mentioned in the contract clause or to invest additional capital in the system affecting in a dramatically the project cost. The second reason is that at this stage normally we see the addition of new distributions with various profiles such as the thermal generation which could stop the gas consumption for a certain time (unballasting). These scenarios must be taken into account since they affect directly the installation plan of the compressor stations and the operational units depending on the service of gas distribution. Moreover, we could use the transient analysis to define beforehand the turbocompressors which match best our system and also define the best arrangement of the units within the station, either in series (some units and larger machines) or in parallel (more units and smaller machines) in a pipeline we would make a failure analysis for only one unit or the complete station and to envisage how the system would behave, determination of gasometric provision, and to define even before the design stage.

\section{System Configuration}

The system to be simulated includes two real networks of different configuration SIMPL1 and SIMPL2.

*Corresponding author: Address: Laboratory of Chemical Engineering, University Ferhat Abbas, Faculty of Engineering, Sétif - Algeria. E-mail : touabtif@yahoo.fr , Phone (213) 73.10.98.06 Faxes (213) 36721787 


\section{$\underline{\text { SIMPL1 }}$}

The system to simulate include a network of gas pipeline of $4000 \mathrm{~km}$ with 400 nodes includes.

(01) Source point

(03) Compression stations (of which one is in project)

(04) Pressure regulators and reducers.

The gas volume is $1,2.10^{9} \cdot \mathrm{m}^{3}$.During summer the uninterrupted deliverable volume raise up to $55010^{6}$ $\mathrm{m}^{3}$ and the maximum instantaneous flow $13 \quad 10^{6}$ $\mathrm{m}^{3} /$ day. Series of flow and pressure measurements were obtained by the means of the SCADA system.

Sections of 125 and $800 \mathrm{~mm}$ are covered internally with an epoxy painting with a roughness of $0.009 \mathrm{~mm}$ and for other non-covered pipelines roughness is $0.018 \mathrm{~mm}$.The pipeline profile varies from the sea level until $(1100 \mathrm{~m})$.

\section{SIMPL2}

The system to simulate include a network of gas pipeline of $750 \mathrm{Km}$ with 200 nodes includes.

(02) Source point

(02) Compression stations

(01) Pressure regulators and reducers.

(01) Underground tank

\section{Importance of Simulations in non-Stationary Modes}

The development of network simulators [2-3] is related to increasing the progress of the computing science and its power. The use of model makes it possible, by simulation, to reach the following objectives:

-To get closer to reality.

-To better manage the transport capacity of the already existing network.

-To better manage gas supply sources of various points of the network.

-To better manage the work of the compression stations.

-To maintain in each critical situation of exploitation, in case of incident, gas supply of the maximum of customers.

\subsection{Transient Analysis}

The principal advantage of this analysis is prediction, to operate and detect any operation problem which could arise [3-4]. A complete transient analysis is carried out starting from the condition of design going back to the year of the first operation.

The performance of the compression stations assigned from the analysis of the stationary state is controlled for each year of operation for the considered scenarios, but now by considering the distribution profiles, in some cases we noticed that the allocation of the station by the stationary state has to be changed to match the transient scenario.

This analysis was extremely important will be also useful during the negotiations of the various contracts, which starts a provision unit for the critical cases.

The transient analysis a better process of maintenance, or even to detect the need for for the project because we would see better in advance the need for capital investment to guarantee transport or to purchase contracts without risk in the future, as well as the definition of the cost of transport involved in the scenarios.

Important assumptions that one must make before any simulation are

- What is the transient event that one studies?

- What is the logical starting point of simulation?

- At which point the system to be described must be detailed?

\section{Case of Model Exploitation}

\subsection{Behaviour and Possibilities of a Network}

To calculate the pressure losses in gas transport network, one uses more often valid simple formulas only in the case of permanent flow mode. Thus, these formulas cannot take into consideration of modes variations caused by the starting or the stop of compressors, closing or the opening of valves or, more simply, by the modulations of consumption at the stations of delivery. Modeling, of the network systems of very complex gas SIMPL 1,2 of configuration in dynamic mode carried out with dynamic simulators (SIMONE 5.53 and TGNet 5.3 ) 


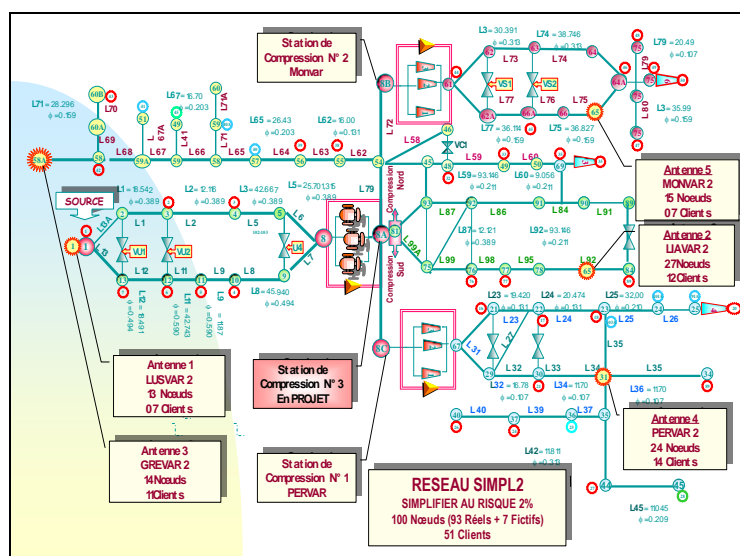

Figure 1 Simulated network SIMPL 1

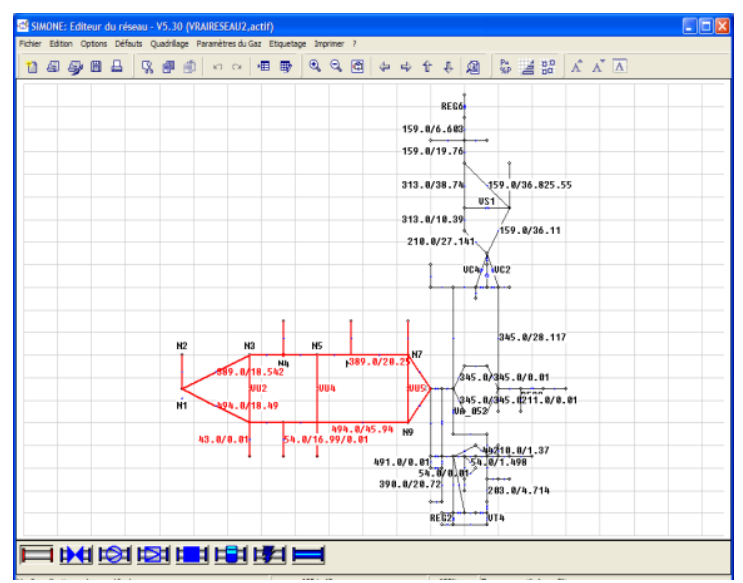

Figure 2 Simulated network SIMPL 1 by SIMONE

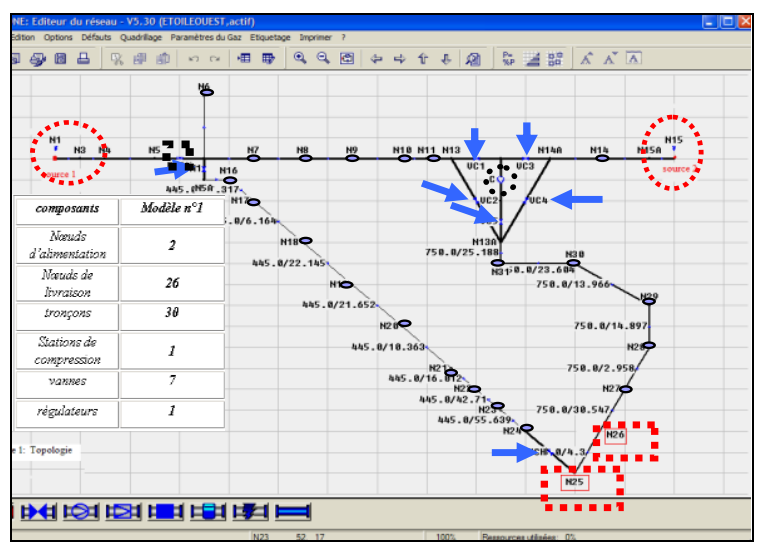

Figure 3 Simulated network SIMPL 2 by Simone

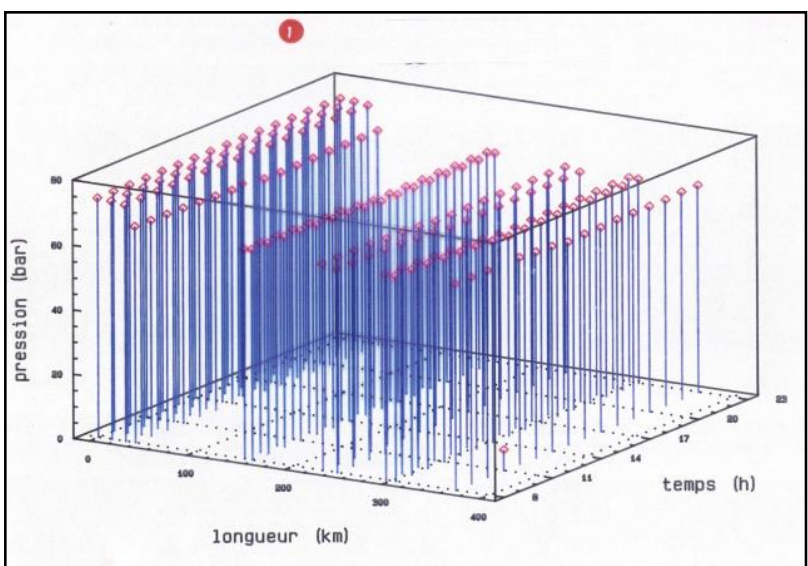

Figure 4 Permanent Flow 3D

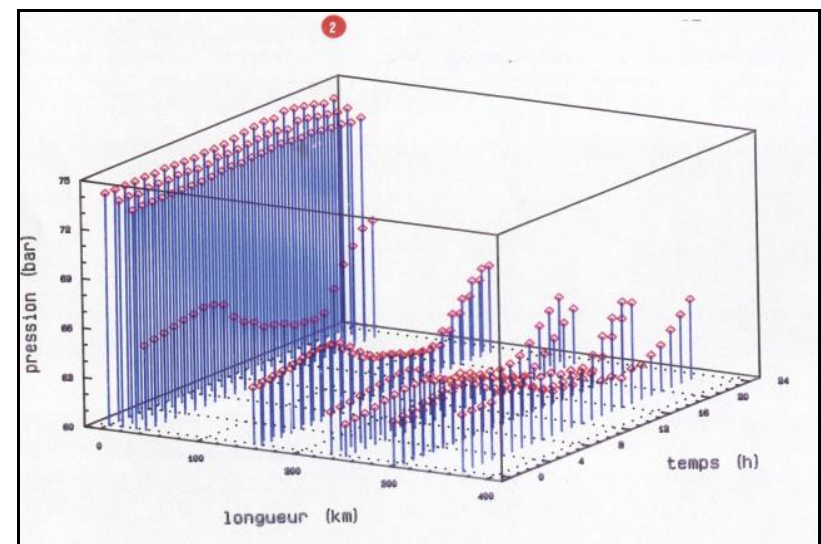

Figure 5 Non-permanent flow 3D (variable)

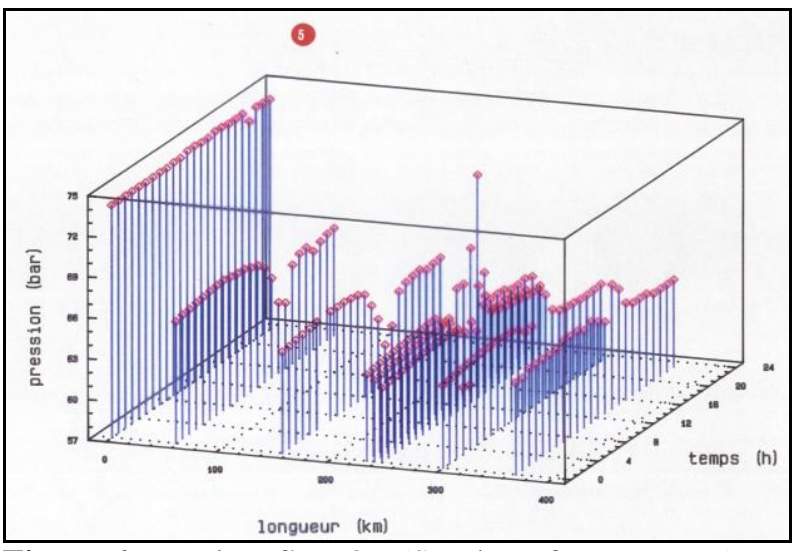

Figure 6 Transient flow 3D (Starting of compressor)

Curves below present:

4. Permanent flow (3D)

5. Variable flow (3D)

6. Transient flow (3D) 


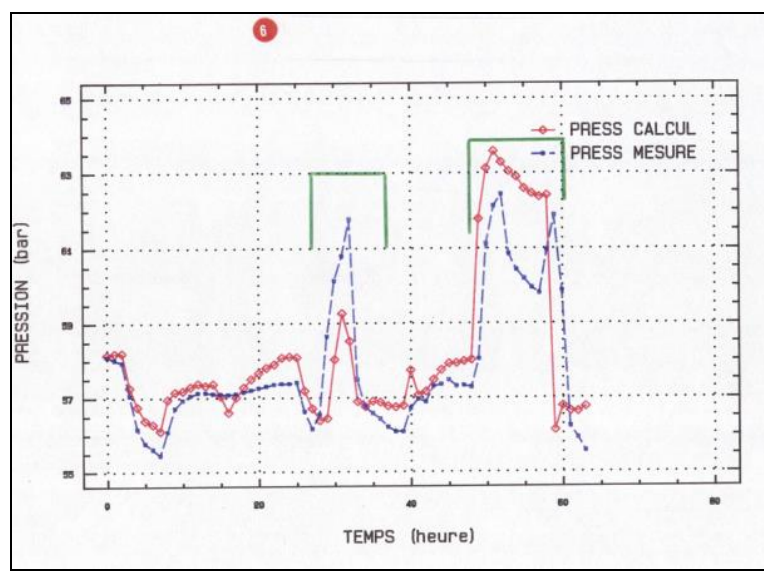

Figure 7 Measured and calculated pressures by TGnet

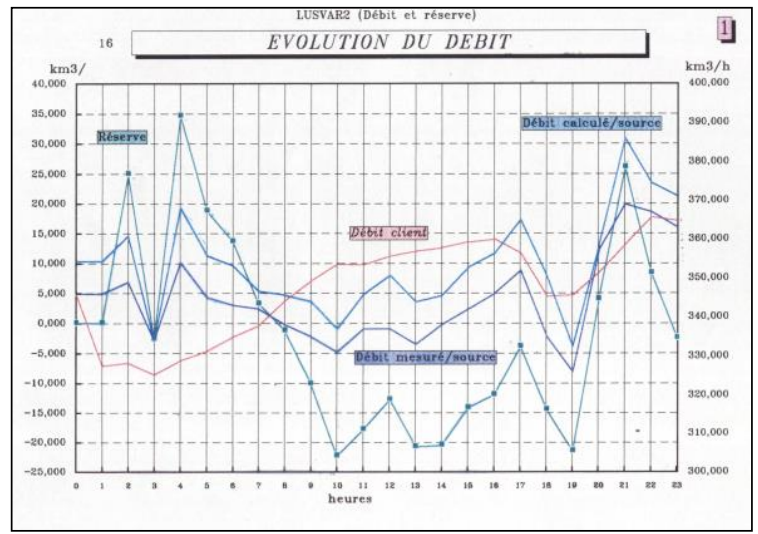

Figure 8 pipe storage capacity by Tgnet

\section{Network Analysis - Check of Validity}

\subsection{Comparison of the Measured and Computed Values}

Simulation in its various forms of the network behaviour allows the study of the flows and pressures evolution in sections. In fact, the network known as "real", limits the code possibilities for a fine description which comprises only a maximum of 100 nodes. We have therefore, on the one hand simplified the network with a maximum of 100 nodes called SIMPL2 (simplified with the risk $2 \%$ ) on the known as "real", limits the code possibilities for a fine description which comprises only a maximum of 100 nodes. We have therefore, on the one hand simplified the network with a maximum of 100 nodes called SIMPL2 (simplified with the risk $2 \%$ ) on the other hand treated, sub-networks of real configuration. Curves of Figure 7 give the variations of measured and computed pressures.
The observed distortions can result:

- Of an insufficient description of the network,

- Of an insufficient description of the scenario

- Of a bad distribution of consumption during the day (over-estimated compared to reality)

- Errors of measurement.

These results show an analysis of the sub-networks, the network and as far as one knows with a good precision the various parameters of study (i.e. the fine description of the network, lengths, diameters, roughnesses and variations of constraints such as consumption and the pressures), the model gives very satisfactory results.

\subsection{A Pipeline Storage Capacity Analysis}

Various treated cases enable us to simulate the behaviour of one pipeline working like a gas reserve and to deduce the role it plays in the lowering of peak demand. Curves of figure 8 give the noted variations of gas volume contained $\mathrm{n}$ the pipeline, flows evolution from the source and at the customer level. This pipeline supplies only an important point of delivery, therefore its use as a reserve must give at the end of the day a nil balance of the assessment variations of volume. Otherwise a positive balance will correspond to the increase of reserve in the pipeline and a negative balance to its reduction.

Figure 8 puts in a prominent position the participation of the pipeline reserve in the lowering of diurnal peaks of consumption.

\section{Transported gas analysis}

Simulation under various hypotheses of the network behaviour allows the study of a section evolution transits. In the present case, we have chosen the subnetwork called "Feeder 5" installed next to the compression station. It is preferable to have a minimum of equipment to provide needed pressures and speed flows since this would decrease the construction and maintenance costs. However, there exist many situations where variable flow effects show a use of equipment different from those of the stationary regime. $\mathrm{n}$ this case, to show variable flow effects, let's consider a single pipeline system of 102 $\mathrm{km}, 159$ and $313 \mathrm{~mm}$ of internal diameter in series. A regulator is placed at the end of this line that gives a constant output pressure of 25 bars. 


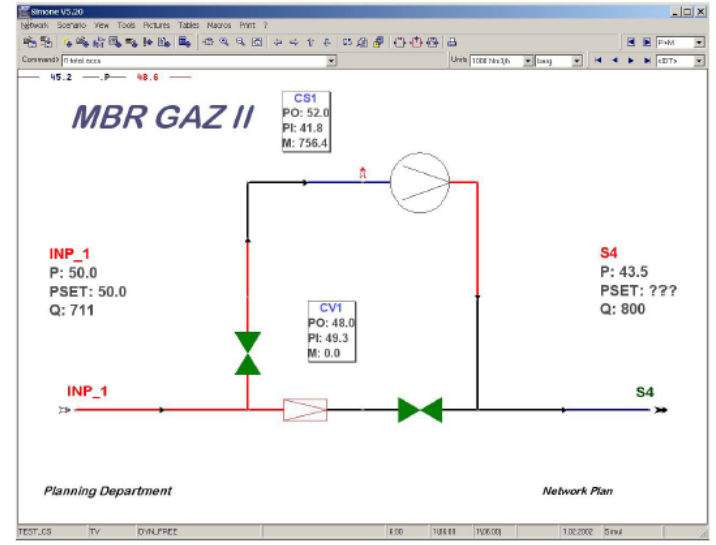

Figure 9 Loops

Let's consider a stationary regime: Pressures and speed flows do not change with time. Let's see now various developments and alternatives that could improve pressure and speed flow conditions, then study each alternative relying on the fact that the regime is not stationary but actually transient.

Stationary regime conditions : The demand predictions reveals that maximum estimated demand at delivery is $807 \mathrm{~km}^{3} /$ day $\left(34 \mathrm{~km}^{3} / \mathrm{h}\right)$ considering that this is the worst case conditions, the stationary regime analysis shows that the predicted delivery pressure is 24 bars. To increase the delivery pressure, some loop cases are considered.

Stationary Regime Loop: A mesh of $76 \mathrm{~km}$ with a pipe of $159 \mathrm{~mm}$ equivalent diameter is suggested. The question is : Would the mesh be placed down or up stream of the system?

The calculations result show that in both the delivery pressure is about 26 bars, taking into consideration that the speed flow of $807 \mathrm{~km}^{3} /$ day remains constant. Knowing that the highest pressures are downstream the pipe, the mesh is placed for the aim of keeping the system reserve of gas at its utmost. To sum up, for a maximum hourly demand of $34 \mathrm{~km}^{3} / \mathrm{h}$, a stationary regime hypotheses show that a mesh of $76 \mathrm{~km}, 159$ $\mathrm{mm}$ diameter placed downstream is needed to ensure a quite good delivery pressure at a minimum of 36 bars.

Transient Analysis: To perform a transient analysis of a system, it is necessary to assess the variable-like nature of the demands in time. A clear definition of the profile demand is a very important aspect in a transient analysis. Figures 11 the resulting pressure profile for a mesh installed at down and up stream.

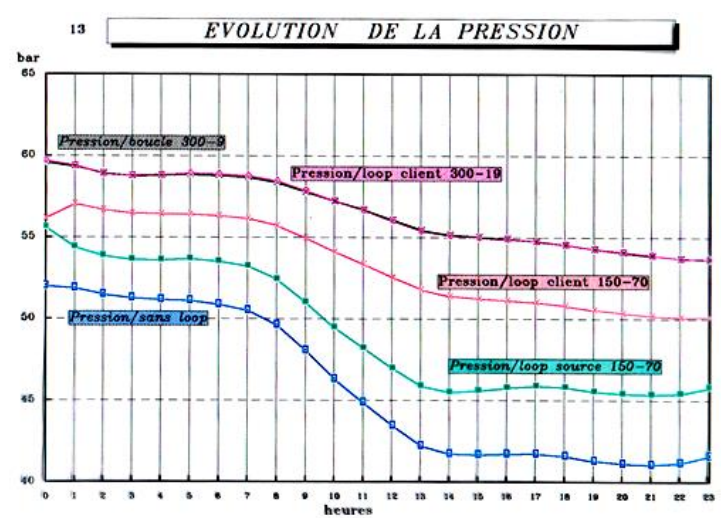

Figure 10 Pressure evolution without compression

These analyses indicates something of a great importance. The stationary regime hypothesis stating that a downstream mesh is more beneficial for delivery pressures proved to be false. Still, it is true that downstream mesh increases the total gas reserve. It becomes clear then that the system is unable to transport the gas up to the delivery point if need be. When we put the mesh closer to the upstream end of the pipe, there follows a reduction of gas reserve but also a higher delivery pressure. The need to study the transport capacity of an existing pipe generally known as "Gas Reserve Management" is required. This study shows the differences between stationary and variable regime analysis.

Whereas the stationary regime shows that a loop is required, the transient analysis shows that the system can sometimes work without any new equipment or installations help. Even if a loop is installed, It remains uncertain that the pressure increase justifies a new installation cost.

\section{Conclusion}

This article, based on real and deepened scenarios pipeline, has as a principal objective to stress the advantages to use transient simulation. Not only like tool but also to highlight its utility in at the stage of design of the gas pipeline.

Dynamic simulators available at the present time are not oriented towards optimization [5, 6, and 7] logic. The simulation techniques allows only to compare alternatives and for this reason to choose the best among which are envisaged. However, efficiency of a complex system, such as an important network of gas pipelines, can depend on hundreds of factors.

Modification of a parameter involves that of the others. This can lead, if one would examine all 
possibilities successively, with a sorting which would require a machine time exceeding the limits of the possible one. It is the principal obstacle of simulation as an optimization instrument of complex systems. In this context, the simulator can be assimilated to an experimentation stand making it possible to test a subset of alternatives and to choose the best combination belonging to this subset.

\section{References}

[1] Larson RE, Wismer D.At 1971 "Hierarchical control of transient flow in natural gas pipeline network "Proc. IFAC symp.control of distributed parameter systems.

[2] Marks D.Morari M.1988 "On-line optimization of gas pipeline network "Automatica, flight.24, $\mathrm{N}^{\circ} 4$
[3] Pascal H, 1970 " Nonpermanent flows in the gas pipelines Edition Technip, Paris

[4] R.Michon-M-Sorine, "Model digital of calculation gas flow in variable mode"

[5] Schmidt G. Weimann A. Lappus G. 1978 "Application of simulation techniques to planning, supervision and control of natural gas distribution network.

[6] Sood AK. Funk GL. Delmastros A. C.1971 " Dynamic optimization of natural gas pipeline using has gradient search technical "Int J. Control, flight.14 $\mathrm{N}^{\circ} 6$.

[7] With Furness -"Modern pipeline monitoring techniques" Pipes\&pipelines international May-June 1985. 